\title{
Gobierno local y desarrollo sustentable. Caso de las ciudades de Oaxaca, México
}

\section{Christian Martínez Olivera}

Estudiante del Doctorado en Ciencias en Desarrollo Regional y Tecnológico en la División de posgrado e Investigación del Instituto Tecnológico de Oaxaca/Tecnológico Nacional de México. ORCID: https://orcid.org/00000002-2564-5038. Maestro en Ciencias en Desarrollo Regional y Tecnológico. Licenciado en Ingeniería Mecánica por el Instituto Tecnológico de Oaxaca. Líneas de investigación: desarrollo regional sustentable, gobernanza local, ciudades, planificación estratégica.

christianolivera26@gmail.com

\section{Andrés Enrique Miguel Velasco}

Profesor investigador en la División de Posgrado e Investigación del Instituto Tecnológico de Oaxaca, Tecnológico Nacional de México. ORCID: https://orcid.org/0000-00031525-5017. Doctor en Ciencias en Planificación de Empresas y Desarrollo Regional del Tecnológico Nacional de México. Maestro en Desarrollo Urbano del Colegio de México A. C. y licenciado en Ingeniería Civil en Desarrollo de la Comunidad del Tecnológico Nacional de México. Su línea de investigación es el desarrollo regional sustentable. andres.miguel@itoaxaca.edu.mx

\section{Karina Aidee Martínez García}

Estudiante del doctorado en Ciencias en Desarrollo Regional y Tecnológico en la División de Posgrado e Investigación del Instituto Tecnológico de Oaxaca, Tecnológico Nacional de México. ORCID: https://orcid.org/0000-00028481-0902. Maestra en Ciencias en Desarrollo Regional y Tecnológico, licenciada en Ingeniería Industrial del Instituto Tecnológico de Oaxaca, Tecnológico Nacional de México. Sus líneas de investigación son el desarrollo regional sustentable y la vivienda con TIC. aidee1005@gmail.com

\section{Julita Moreno Avendaño}

Doctora y Maestra en Ciencias en Desarrollo Regional y Tecnológico por el Instituto Tecnológico de Oaxaca, Tecnológico Nacional de México. Ingeniera industrial por la misma institución. Miembro del grupo de investigación de ciudades en la División de Estudios de Posgrado del Instituto Tecnológico de Oaxaca. ORCID: https://orcid.org/0000-00017482-8159. Sus líneas de investigación son desarrollo regional sustentable, desigualdad urbana y territorial, y pobreza.

julitamorenoave@gmail.com 


\section{Gobierno local y desarrollo sustentable. Caso de las ciudades de Oaxaca, México}

\section{Resumen}

El presente artículo tiene como objetivo conocer el manejo de los ingresos municipales por parte de los gobiernos locales y su impacto en el desarrollo sustentable de las ciudades. La hipótesis que propone es que el uso de los ingresos municipales tiene un impacto positivo y significativo en el desarrollo sustentable. Se analizan diecisiete ciudades del estado de Oaxaca, México, durante 2000-2017. Aplicando una metodología cuantitativa con herramientas de estadística descriptiva y regresión, se obtiene una alta asociación entre el desarrollo sustentable y la gestión del gobierno local, lo que indica que el manejo adecuado de los ingresos municipales por parte de los gobiernos locales tiende a influir favorablemente en el desarrollo sustentable de las ciudades de estudio. Por esta razón son un elemento primordial para revertir los escenarios desfavorables a nivel global y favorecer la recuperación de la dinámica del desarrollo de las ciudades.

\section{Palabras clave}

Gobierno local; desarrollo sustentable; ciudades; ingresos municipales.

\section{Local government and sustainable development. The case of Oaxaca's cities, Mexico}

\begin{abstract}
The objective of this article is to know about the management of municipal revenues by local governments and its impact on the sustainable development of cities. The hypothesis it proposes is that the use of municipal revenues has a positive and significant impact on sustainable development. Seventeen cities of the state of Oaxaca, Mexico, during 2000-2017 are analyzed. Applying a quantitative methodology with descriptive statistics and regression tools, a high association between sustainable development and local government management, which indicates that the proper management is obtained, which indicates that the adequate management of municipal revenues by local governments tends to have a favorable influence on the sustainable development of the cities under study. For this reason, they are an essential element to revert the unfavorable scenarios at a global level and to favor the recovery of the cities' development dynamics.
\end{abstract}

\section{Keywords}

Local government; sustainable development; cities; municipal revenue.

\section{Governo local e desenvolvimento sustentável. Caso das cidades de Oaxaca, México}

\section{Resumo}

O objetivo deste artigo é conhecer a gestão das receitas municipais pelos governos locais e o seu impacto no desenvolvimento sustentável das cidades. A hipótese proposta é que o uso das receitas municipais tem um impacto positivo e significativo no desenvolvimento sustentável. Dezessete cidades do estado de Oaxaca, México, durante 2000-2017 são analisadas. Aplicando uma metodologia quantitativa com estatística descritiva e ferramentas de regressão, obtém-se uma alta associação entre o desenvolvimento sustentável e a gestão dos governos locais, o que indica que a gestão adequada das receitas municipais pelos governos locais tende a influenciar favoravelmente no desenvolvimento sustentável das cidades em estudo. Por esta razão, são um elemento essencial para reverter os cenários desfavoráveis a nível global e favorecer a recuperação da dinâmica do desenvolvimento das cidades.

\section{Palavras chave}

Governo local; desenvolvimento sustentável; cidades; receita municipal. 


\section{Introducción}

La falta de planificación y el progresivo crecimiento de las ciudades latinoamericanas, sumados a la complejidad político-social, ambiental y económico-global, muestran un escenario socioeconómico poco alentador en los años venideros para mejorar el desarrollo sustentable. Sobre todo porque el actual momento de incertidumbre a consecuencia del problema mundial de salud de la COVID-19 ha puesto en jaque a los gobiernos nacionales. De acuerdo con el Banco Mundial (2020), se avecina la que sería la peor recesión desde la Segunda Guerra Mundial, y la primera vez desde 1870 en que tantas economías experimentarían una disminución de su producto per cápita. La pregunta al respecto es: ¿qué tanto pueden influir los gobiernos locales para revertir este escenario global desfavorable para el desarrollo sustentable de las ciudades?

Por esta razón, el propósito del presente artículo es conocer la dinámica del desarrollo sustentable de las ciudades que cuentan con población mayor a 15.000 habitantes, para determinar cuánto ha influido el gobierno local, a través del uso de los ingresos municipales y su respectiva incentivación, destacando que los gobiernos locales tienen un papel importante en la resistencia y acción inmediata ante las crisis globales. Con base en esta preocupación, en el presente artículo se analizan diecisiete ciudades del estado de Oaxaca, México, durante 2000-2017, señaladas en la figura 1. Se propone como hipótesis que, durante el período considerado se genera una asociación positiva entre la gestión de los gobiernos locales y los niveles de desarrollo sustentable alcanzado por las ciudades; es decir, la gestión gubernamental a través del manejo adecuado de los ingresos municipales influye favorablemente en el desarrollo sustentable de las ciudades en estudio.

\section{Eje teórico: desarrollo sustentable y gobierno local}

\subsection{La relación ciudad-gobierno local}

La actuación del gobierno local es clave para potencializar las oportunidades y fortalezas con las que cuentan las ciudades, así como dinamizar su desarrollo, considerando que una ciudad, según CASADO (2010, p. 4), es el "centro territorial donde se acumula el capital”, también funge "como instrumento colectivo de la reproducción social, y se considera que es la mejor forma de usar el espacio desde un aspecto económico, siendo el centro de comercialización en las diversas regiones”. Al respecto, para algunos autores entender la dinámica 
de la ciudad es un tema complejo que se mira desde diferentes perspectivas objetivas y subjetivas (GARCíA, 2016; LóPEZ ET AL., 2018). En la búsqueda de soluciones para mejorar la economía de la ciudades, se hace alusión a la incorporación del conocimiento y la creatividad por medio de las ideas de sus habitantes, para dinamizar y transformar las zonas urbanas con base en la organización político-social que promueve el desarrollo (FLoRIDA, 2005; ScotT, 2007), cuestiones de gran importancia para el apoyo de toma de decisiones en los distintos aspectos que impactan en el bienestar de las ciudades. No obstante, para M outinHo y FARIA (2019) y ToRíbio (2013), existen tres principales retos para las ciudades: falta de igualdad, equidad y resiliencia; debilidad de la gobernabilidad local y participación ciudadana y el nulo reconocimiento de la cultura en la formulación de políticas públicas de gestión ambiental como pilar básico del desarrollo sustentable.

Al respecto, resalta la importancia de los gobiernos locales y su capacidad de planificación del territorio, siendo la institución gubernamental más cercana y susceptible de ser receptiva a

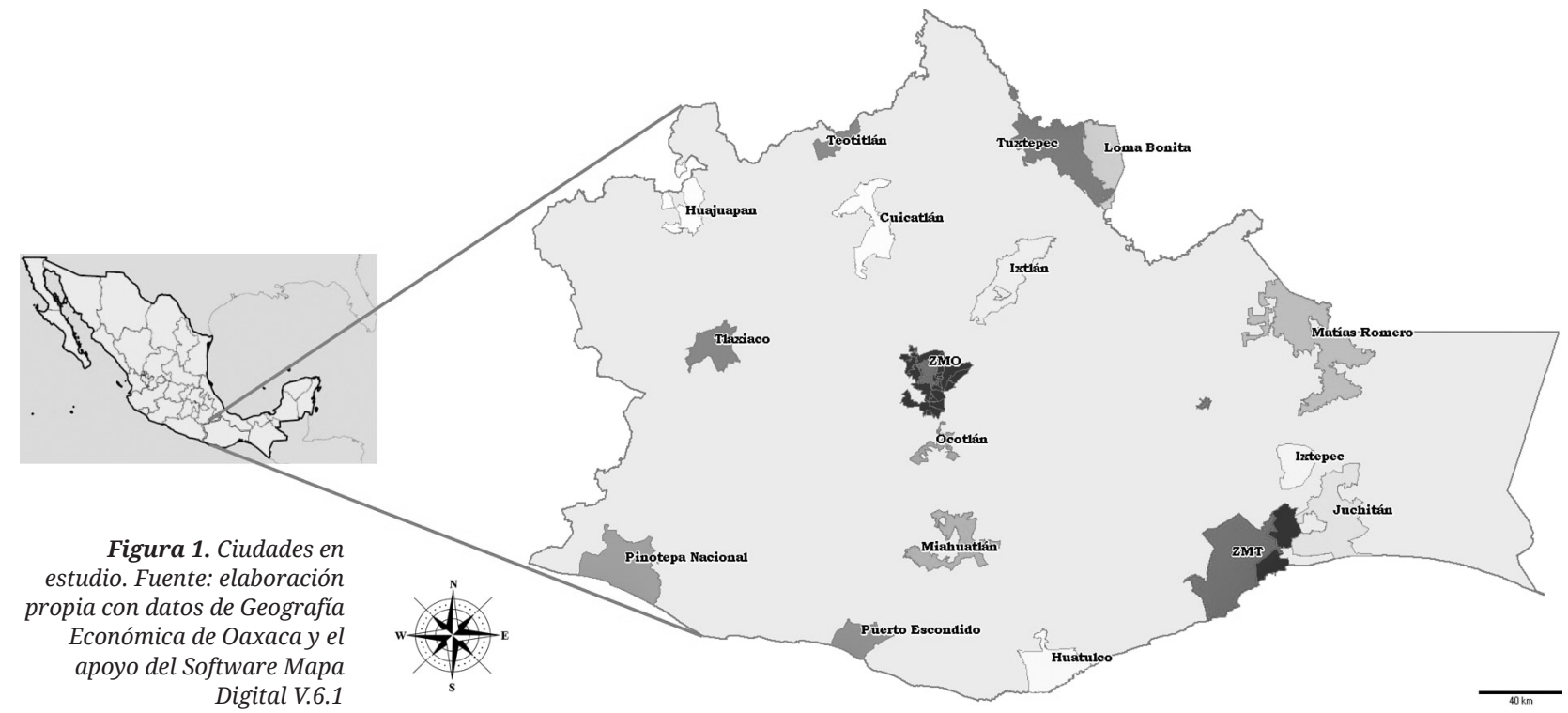


la voz de los ciudadanos y sus demandas, entre otras, con la necesidad de conseguir calidad gubernamental urbana, que para URIBE (2020) se refiere a propiciar las condiciones satisfactorias con parámetros óptimos de la habitabilidad para el espacio ocupado, es decir, a lo edificado y su entorno: el área de espacio público, servicios domiciliarios, vivienda, entre otros.

Como sugieren López y Soria (2013), una manera de obtener calidad en los resultados de los gobiernos locales es a través de la gestión pública y su modelo operativo para el sector público con el objetivo de que funcione mejor. De acuerdo con KEN (2014), es un conjunto de metodologías, técnicas y procesos que conducen a la mejoría del nivel de vida de los habitantes, incluyendo actividades de seguimiento, transparencia y evaluación. Este tipo de herramienta nace de la necesidad de profesionalizar servidores públicos con ética y compromiso social para mejorar su desempeño público. Asimismo, constituye una oportunidad para reconocer a los gobiernos locales como sujetos activos de cooperación y desarrollo, teniendo como medio de fortalecimiento institucional el proceso de intercambio de experiencias participando en redes internacionales, para dar respuestas locales a problemas globales con el protagonismo de la ciudadanía en la toma de decisiones (SÁnchez \& MARTínez, 2016).

En muchos casos, los distintos intereses y la resistencia al cambio son obstáculos para el desarrollo de las ciudades. En tal sentido, SÁNchEZ ET AL. (2018) argumentan que las costumbres viciadas, como considerar que el desarrollo de los territorios es exclusivo de las intervenciones del gobierno central y que su participación en la dimensión de políticas públicas es complementaria y acotada, aumentan el conformismo del gobierno local. A pesar de lo anterior, en la última década se han dado transformaciones de descentralización en las estructuras políticas hacia nuevos escenarios y actores, que otorgan a la sociedad mayor posibilidad de ejercer su poder público (EspinozA, 2017). Además, a través de un trabajo en red con otros gobiernos locales, se han dado nuevas relaciones y formas de gobernabilidad de los territorios en la operación y ejecución de las políticas públicas, que agregaron valor y productividad (VARELA, 2015). En otras palabras, las viejas prácticas y estructuras de las instituciones locales se han visto mermadas, y se abrió paso a una nueva forma de gobernar localmente.

En resumen, crear un escenario adecuado para las ciudades con servicios e infraestructuras urbanas eficientes y buena calidad de vida en general requiere la colaboración entre el sector público, el privado y la sociedad. Implica, además, la modificación del ambiente gubernamental dentro del contexto de la interconexión interna y externa, pensando en 
forma "glocal", es decir, tomando en cuenta lo que suceda en lo global para tomar acciones en lo local (Salvatro \& Banzato, 2013; DAmmert, 2007). Por tal razón, la relación entre la ciudad y el gobierno local es clave para potencializar las oportunidades y fortalezas con el fin de dinamizar su desarrollo.

\subsection{La relación gobierno local-desarrollo sustentable}

En la mayor parte de los países latinoamericanos, la capacidad de los gobiernos locales para planificar su territorio está limitada a consecuencia de factores como la insuficiencia de recursos presupuestales y técnicos (López ET AL., 2017). En México, de acuerdo con Carmona y CAAMAL (2018), la dependencia financiera es el principal factor de estancamiento del desarrollo en los territorios, siendo las transferencias federales la más importante fuente de recursos con que cuentan los municipios. En temas como la recaudación y transparencia fiscal, sitúa al gobierno local en un nuevo escenario, en particular, con la necesidad de utilizar los recursos con los que cuenta para propósitos generales sin condicionamiento y con destino de gasto específico.

Tomando en cuenta que el desarrollo sustentable "está comprendido por la sociedad, las estructuras económicas y los sistemas ambientales en los que se desenvuelve, orientado a la elevación del bienestar" (Miguel, 2012, p. 72), se ha convertido en una necesidad para enfrentar los problemas de las ciudades, involucrando un proceso de transformación que busca hacer uso de los recursos endógenos para recrear un entorno institucional, político, económico y cultural de fomento de las actividades productivas en los diferentes ámbitos territoriales (Ionita et al., 2011). Su principal peculiaridad radica en que las condiciones histórico-concretas de cada lugar configuran el modelo de desarrollo sustentable; es decir, no puede existir un modelo idéntico en todos los territorios, pero sí con un mismo fin. Lo anterior ubicando en el centro del proceso a los actores y agentes del desarrollo, otorgándoles valor y respeto a sus propuestas y críticas (López \& CHAucA 2010).

En una época en que se privilegia lo económico olvidando los límites ambientales, y ante la emergencia actual, el desarrollo local debe ampliar su significado y pasar de una visión restringida a otra ampliada de calidad de vida y desarrollo respetuoso del entorno ambiental (GuTiÉrREZ \& GonZÁLEZ, 2010; VARELA, 2015). Se habla entonces del desarrollo sustentable como el crecimiento de un territorio, capaz de lograr que la producción y el consumo se fundamenten en una nueva conducta que garantice el bienestar social, económico, 
político-administrativo, cultural y ambiental de las generaciones actuales, sin afectar la igualdad de desarrollo de las futuras generaciones (HERNÁndEZ ET AL., 2017). Los gobiernos locales deben promover mejores estrategias de planificación local con los ingresos financieros con que cuentan, sean federales y propios, aprovechando el recurso inagotable de la creatividad que la ciudadanía puede aportar (Pérez \& ArenAs, 2012). Esto dará como resultado ciudades con mejores niveles de vida, a través de un desarrollo sustentable alcanzable y con una acertada gestión local en que la participación ciudadana es fundamental (COSUDE, 2019).

En suma, el desarrollo sustentable tiende a ser el reflejo del actuar de los gobiernos locales, los cuales son uno de los principales entes para la consecución de resultados. Estos tienen la responsabilidad social de utilizar los ingresos municipales de forma eficiente, implementando estrategias para crear las condiciones necesarias y generar un óptimo desarrollo sustentable. Se espera entonces que exista una asociación positiva entre la gestión de los gobiernos locales y los niveles de desarrollo sustentable alcanzados por las ciudades; es decir, con una mejor gestión a través del manejo adecuado de los ingresos municipales, se esperaría un aumento de los niveles de desarrollo sustentable de las ciudades.

\section{Procedimiento metodológico}

Para el presente estudio, se eligieron las ciudades (figura 1) contempladas como el municipio en conjunto; en la tabla 1 se indican su nombre oficial y el nombre cotidiano y sus abreviaturas, con las que se identificarán a partir de este punto, algunas características geográficas y los criterios utilizados para su elección. El análisis tiene un enfoque cuantitativo, siendo del tipo correlacional y explicativo, partiendo del supuesto de que, durante el período de estudio, el uso de los ingresos municipales por parte del gobierno local tiene un impacto significativo en el desarrollo sustentable de las ciudades analizadas.

Con respecto al modelo metodológico, en el análisis se consideran dos variables: como variable independiente el índice de ingresos municipales (I_IMUN), que mide el nivel de ingresos en cada una de las ciudades considerando ingresos ordinarios y federales. Como variable dependiente, el índice de desarrollo sustentable (I_IDS), que incluye aspectos económicos, sociales, ambientales y territoriales. Dicho índice trata de medir el grado de sustentabilidad a nivel local de las ciudades consideradas (tabla 2). Estos índices fueron diseñados por medio de la media geométrica de cada uno de sus componentes. Para el análisis y unificación 
de los datos, se efectúa una estandarización de acuerdo con la metodología de SEPÚLVEDA (2008, p. 32), según la cual Ic: índice del componente; VR: valor real del indicador; VMÁX: valor máximo del indicador; VMíN: valor mínimo del indicador (ecuación 1).

$$
\text { Ic }=\frac{(V R-V M I ́ N)}{(V M A ́ X-V M I ́ N)}
$$

Tabla 1

\begin{tabular}{|c|c|c|c|c|c|c|c|}
\hline \multirow[b]{2}{*}{ Nombre Oficial } & \multirow[b]{2}{*}{$\begin{array}{l}\text { Nombre } \\
\text { cotidiano }\end{array}$} & \multirow[b]{2}{*}{ Abrev. } & \multirow[b]{2}{*}{ Población } & \multicolumn{3}{|c|}{ Coordenadas geográficas } & \multirow[b]{2}{*}{$\begin{array}{l}\text { Criterios de } \\
\text { elección }\end{array}$} \\
\hline & & & & $\begin{array}{l}\text { Latitud } \\
\text { norte }\end{array}$ & $\begin{array}{l}\text { Longitud } \\
\text { oeste }\end{array}$ & $\begin{array}{l}\text { Altitud } \\
\text { msnm }\end{array}$ & \\
\hline Ixtlán de Juárez & Ixtlán & IXN & 8268 & $17^{\circ} 19^{\prime} 50^{\prime \prime}$ & $96^{\circ} 29^{\prime} 14^{\prime \prime}$ & 2,030 & \multirow{17}{*}{$\begin{array}{c}\text { De "elegibili- } \\
\text { dad": que sean } \\
\text { lugar central } \\
\text { de una región } \\
\text { socioeconómica } \\
\text { estatal, basada } \\
\text { en una regionali- } \\
\text { zación mixta con } \\
\text { una población } \\
\text { a partir de los } \\
8000 \text { hab. } \\
\text { De "exclusión”, } \\
\text { que no sean } \\
\text { lugar central } \\
\text { de una región } \\
\text { socioeconómica, } \\
\text { y con población } \\
\text { menor a los } 8000 \\
\text { hab. }\end{array}$} \\
\hline San Juan Bautista Cuicatlán & Cuicatlán & CUI & 9945 & $17^{\circ} 47^{\prime} 55^{\prime \prime}$ & $96^{\circ} 57^{\prime} 35^{\prime \prime}$ & 620 & \\
\hline Teotitlán de Flores Magón & Teotitlán & TEO & 9876 & $18^{\circ} 07^{\prime} 57^{\prime \prime}$ & $97^{\circ} 04^{\prime} 20^{\prime \prime}$ & 1,067 & \\
\hline Ciudad Ixtepec & Ixtepec & IXP & 22.675 & $16^{\circ} 33^{\prime} 46^{\prime \prime}$ & $95^{\circ} 06^{\prime} 00^{\prime \prime}$ & 61 & \\
\hline Loma Bonita & Loma Bonita & LOB & 40.877 & $18^{\circ} 06^{\prime} 25^{\prime \prime}$ & $95^{\circ} 52^{\prime} 50^{\prime \prime}$ & 30 & \\
\hline Matías Romero Avendaño & Matías Romero & MAR & 40.709 & $16^{\circ} 52^{\prime} 20^{\prime \prime}$ & $95^{\circ} 02^{\prime} 30^{\prime \prime}$ & 198 & \\
\hline Miahuatlán de Porfirio Díaz & Miahuatlán & MIA & 32.555 & $16^{\circ} 19^{\prime} 42^{\prime \prime}$ & $96^{\circ} 35^{\prime} 46^{\prime \prime}$ & 1,558 & \\
\hline Ocotlán de Morelos & Ocotlán & OCO & 18.183 & $16^{\circ} 47^{\prime} 29^{\prime \prime}$ & $96^{\circ} 40^{\prime} 30^{\prime \prime}$ & 1,513 & \\
\hline Puerto Escondido & Puerto & PES & 32.471 & $15^{\circ} 51^{\prime} 43^{\prime \prime}$ & $97^{\circ} 04^{\prime} 18^{\prime \prime}$ & 65 & \\
\hline Heroica Ciudad de Tlaxiaco & Tlaxiaco & TLX & 29.026 & $17^{\circ} 16^{\prime} 10^{\prime \prime}$ & $97^{\circ} 40^{\prime} 45^{\prime \prime}$ & 2,063 & \\
\hline Crucecita & Huatulco & CRU & 28.327 & $15^{\circ} 46^{\prime} 08^{\prime \prime}$ & $96^{\circ} 08^{\prime} 06^{\prime \prime}$ & 35 & \\
\hline Santiago Pinotepa Nacional & Pinotepa & PIN & 44.193 & $16^{\circ} 20^{\prime} 17^{\prime \prime}$ & $98^{\circ} 03^{\prime} 01^{\prime \prime}$ & 199 & \\
\hline $\begin{array}{l}\text { Heroica Ciudad } \\
\text { de Huajuapan de León }\end{array}$ & Huajuapan & HJP & 53.219 & $17^{\circ} 48^{\prime} 14^{\prime \prime}$ & $97^{\circ} 46^{\prime} 33^{\prime \prime}$ & 1,584 & \\
\hline $\begin{array}{l}\text { Heroica Ciudad } \\
\text { de Juchitán de Zaragoza }\end{array}$ & Juchitán & JUC & 78.512 & $16^{\circ} 26^{\prime} 00^{\prime \prime}$ & $95^{\circ} 01^{\prime} 10^{\prime \prime}$ & 20 & \\
\hline San Juan Bautista Tuxtepec & Tuxtepec & TUX & 133.913 & $18^{\circ} 05^{\prime} 10^{\prime \prime}$ & $96^{\circ} 07^{\prime} 26^{\prime \prime}$ & 20 & \\
\hline $\begin{array}{l}\text { Zona Metropolitana } \\
\text { de Tehuantepec }\end{array}$ & ZMT & ZMT & 145.567 & $16^{\circ} 19^{\prime} 28^{\prime \prime}$ & $95^{\circ} 14^{\prime} 27^{\prime \prime}$ & 44 & \\
\hline Zona Metropolitana de Oaxaca & ZMO & ZMO & 501.283 & $17^{\circ} 3 ’ 55^{\prime \prime}$ & $96^{\circ} 43^{\prime} 25^{\prime \prime}$ & 1567 & \\
\hline
\end{tabular}

Fuente: Tomado de MARTíNEZ (2018), con base al Sistema de Información Municipal (INAFED, 2019) 
Gobierno local y desarrollo sustentable.

Caso de las ciudades de Oaxaca, México

Tabla 2

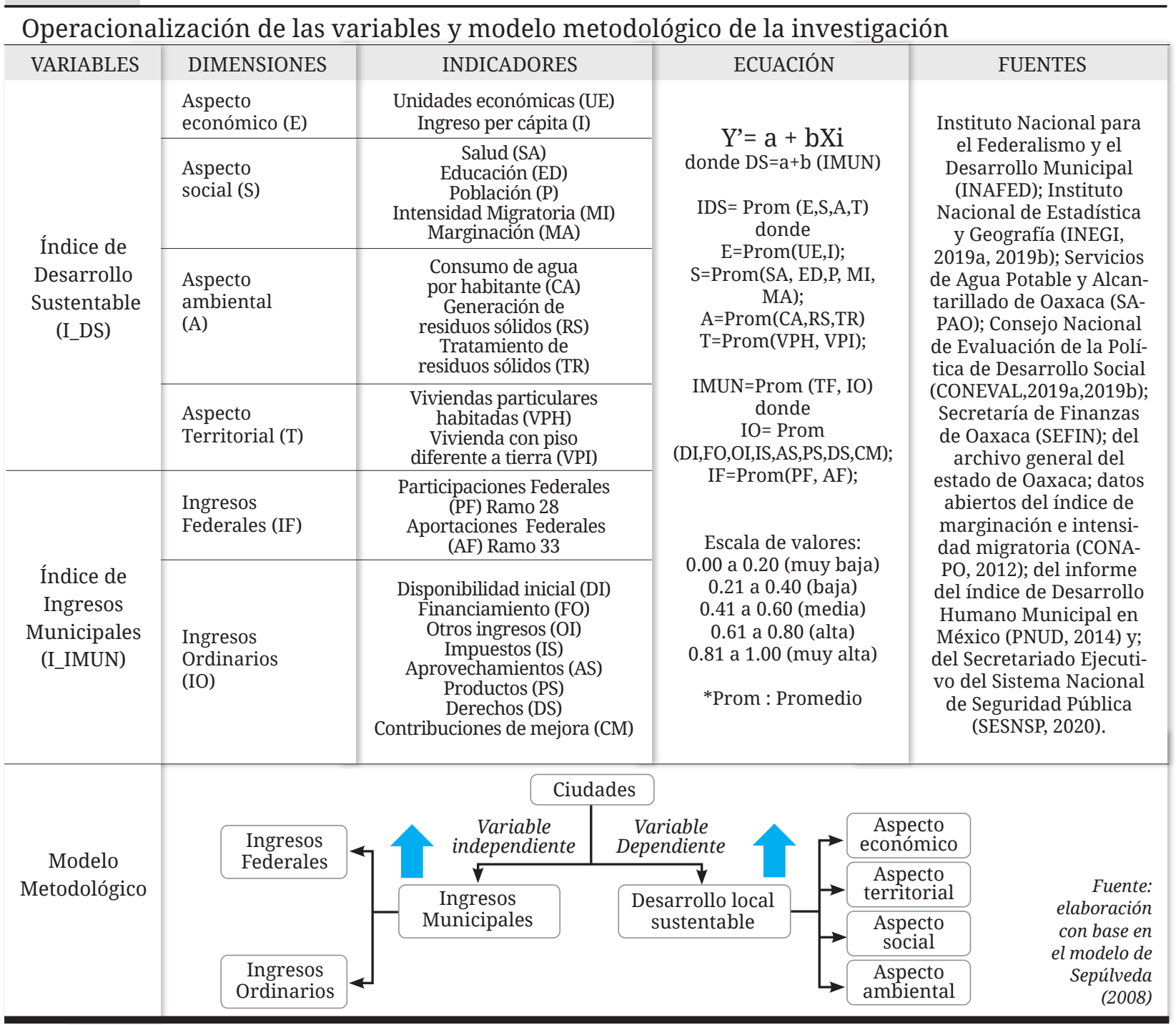


En cuanto a la construcción de los indicadores (I_DS e I_IMUN), su diseño fue de acuerdo con una base de datos conformada por la recopilación de información documentada de instituciones públicas. La operacionalización planteada en la tabla 2 con respecto a los índices permite medir los grados de desarrollo regional y de los ingresos municipales obtenidos durante el período en cada una de las ciudades en estudio. Estos se determinan mediante los valores relativos de sus indicadores, con las fórmulas que constituyen las dimensiones correspondientes al I_DS e I_IMUN, que se evalúan con valores estandarizados con la escala de $0.00 \mathrm{a}$ 0.20 (muy baja), 0.21 a 0.40 (baja), 0.41 a 0.60 (media), 0.61 a 0.80 (alta) y 0.81 a 1.00 (muy alta).

En la tabla 3 se muestran los resultados del análisis de regresión lineal realizado. Para evaluar la relación entre los indicadores y así proceder a la construcción de los índices del artículo que se utiliza para cada una de las variables correspondientes, se aplicó la prueba KMO. Se considera que la medida de adecuación estadística muestral KMO varía entre 0 y 1 ; el resultado obtenido es aceptable al tener un valor de 0.5 .

Para la comprobación de la hipótesis se utilizaron herramientas estadísticas, en este caso el programa estadístico IBM SPSSv21 para la regresión y con el fin de obtener la relación existente entre el I_IMUN y el I_DS. Además, los resultados del análisis se aceptan siempre y cuando se cumplan los siguientes parámetros: si es mayor a 0.60 con una significancia menor o igual a 0.05. La relación que se expone deriva del análisis de regresión lineal que se aprecia en la ecuación uno, donde: I_DS: valor predictivo de la variable dependiente; a: intersección de la línea recta con el eje Y; b: pendiente de la línea recta; I_IMUN: valor de la variable independiente (ecuación 2).

$$
\text { I_DS= a + b I_IMUN-------(2) }
$$

En la tabla 3 se muestran los parámetros del modelo matemático entre el I_DS e I_IMUN, observando una asociación entre las variables analizadas, que es alta y significativa (coef. corr. 0.957; sig. 0.000).

\section{Resultados}

El escenario actual para las ciudades es complicado en los aspectos socioeconómico, ambiental, territorial y, sobre todo, social. El reto para la recuperación será cuesta arriba, 
y se tardará al menos un par de años en volver a la dinámica que se tenía antes de la crisis sanitaria. En consecuencia, se necesitará la participación de todos los sectores, principalmente del gobierno local en lo que respecta a una planificación apropiada de las ciudades, así como el manejo adecuado de los ingresos municipales que tengan a su disposición. También será indispensable la creatividad que puedan aportar los ciudadanos para utilizar las potencialidades de su territorio y contribuir a reducir el tiempo de recuperación de la dinámica económica.

Al respecto, el estado de Oaxaca tiene como uno de sus mayores potenciales su diversidad cultural y gastronómica, aspectos que lo posicionan en el mapa turístico mundial. En el caso de las ciudades analizadas en el presente estudio, todas cuentan con características que contribuyen a fomentar el turismo comercial y el turismo rural en sus territorios. Principalmente la zona metropolitana de Oaxaca, Huatulco y Puerto Escondido, puesto que son las ciudades que concentran un porcentaje turístico alto en comparación con el resto del estado. Desafortunadamente, ante la situación actual, el sector turístico ha sido uno de los más golpeados a nivel mundial. Sin embargo, es precisamente ante este escenario cuando la creatividad en combinación con la participación gubernamental local es clave para el diseño de estrategias para renovar y adaptar la dinámica económica a las necesidades de seguridad y confianza en materia de bioseguridad. De no darse esta combinación, por más diversidad cultural con la que se cuente, un escenario probable es seguir en el estancamiento económico, y, en consecuencia, la recuperación de las ciudades será aún más prolongada.

En esta línea, en la figura 2 se observa la dinámica del comportamiento de los índices que conforman el desarrollo sustentable y de los ingresos municipales de las ciudades de estudio. En el gráfico 2a, se aprecia el comportamiento de las dimensiones del desarrollo sustentable, en el cual durante el período en estudio fueron los aspectos ambiental y social los que contaron con buenos resultados en la mayoría de las ciudades, resaltando la ciudad pequeña de Ixtlán y las ciudades medianas de Tlaxiaco y Loma Bonita. La ZMO, la ciudad más grande analizada, fue la que mostró indicadores desfavorables en estos aspectos. Por el contrario, en lo económico y territorial fue precisamente la que sobresalió del resto de las ciudades.

La figura $2 \mathrm{~b}$ muestra el índice de desarrollo sustentable (I_DS), en el cual las zonas metropolitanas de Oaxaca (ZMO) y Tehuantepec (ZMT), Huajuapan y Huatulco son las que obtuvieron los resultados más favorables. En contraparte, Ixtepec, Tuxtepec, Juchitán y Cuicatlán fueron las ciudades que manifestaron bajos resultados. No obstante, y a excepción de la 
ZMO, ninguna ciudad logró que su indicador estuviera por encima del 0.500, es decir, de la media. Esto es una manifestación clara de que los resultados obtenidos en la mayoría de las ciudades son apenas aceptables, pero no lo deseable.

Con respecto a los ingresos municipales, se aprecia cómo en la mayoría de las ciudades de estudio - exceptuando a Ixtlán, Huatulco y Huajuapan- el mayor porcentaje de sus

a) Componentes del Desarrollo Sustentable

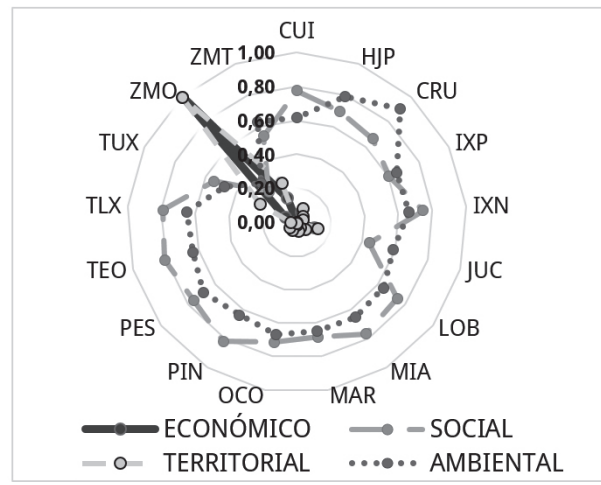

c) Ingresos municipales

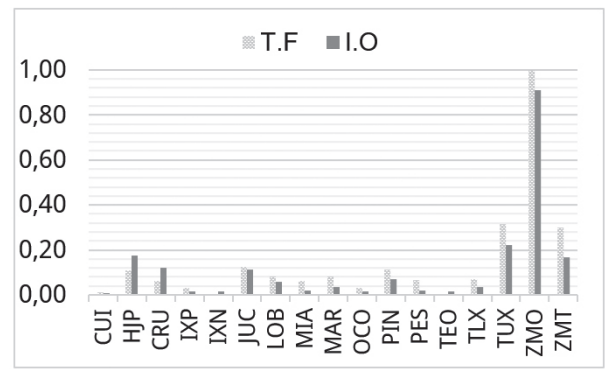

\section{b] Indicador de Desarrollo Sustentable (I_DS)}

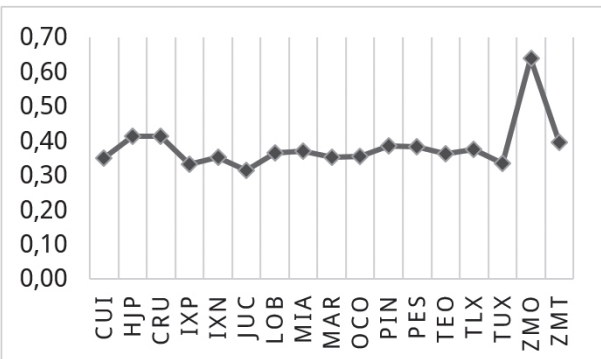

d) Indicador de ingresos Municipales (I_IMUN]

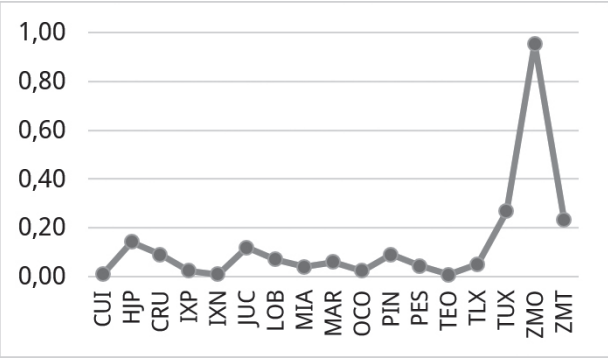

Figura 2. Comportamiento del Desarrollo Sustentable y los Ingresos Municipales en las ciudades en estudio. Fuente: elaboración propia con base en las fuentes de información mostradas en la tabla 2 
ingresos obtenidos durante el período en estudio fueron las transferencias federales (TF), lo que demuestra la debilidad en lo que a recaudación fiscal local e ingresos ordinarios (IO) se refiere (figura 2c). Por otra parte, las ciudades con un índice de ingresos municipales (I_IMUN) alto son la ZMO, ZMT, Tuxtepec y Huajuapan. En contraparte, Cuicatlán, Ixtepec, Ixtlán, Teotitlán del Camino y Ocotlán son las que contaron con menores ingresos. No obstante, y al igual que en el índice de desarrollo sustentable, y exceptuando a la ZMO, ninguna ciudad logró superar los 0.500, es decir, los ingresos con los que contaron, considerando las transferencias federales y los ingresos ordinarios, fueron bajos (figura $2 \mathrm{~d}$ ).

La figura 3 muestra la relación entre el índice del ingreso municipal y el índice del desarrollo sustentable de las ciudades de Oaxaca. Específicamente, la ZMO mostró un índice de ingresos municipales por encima del índice de desarrollo sustentable, pero a pesar de que fue la ciudad que contó con mayores ingresos, estos no se vieron reflejados en su desarrollo sustentable como en el resto, aunque el valor de su índice de desarrollo sustentable fue el más alto de las ciudades y el único que superó la media (0.500). En el resto de las ciudades, el manejo de los ingresos municipales relativamente bajos por parte de los gobiernos locales manifestó un impacto positivo proporcional medio para el desarrollo de las ciudades.

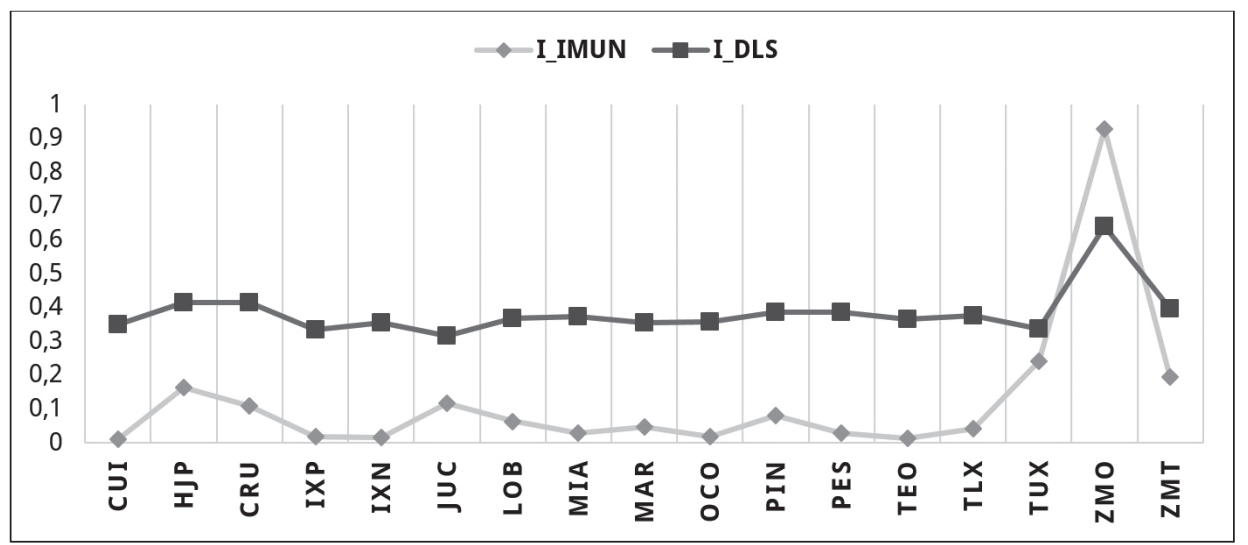

Figura 3. Relación entre el Ingreso Municipal (I_IMUN) y el Índice de Desarrollo Sustentable (I_DS) Fuente: elaboración propia con base en las fuentes de información mostradas en la tabla 2 
Tabla 3

\section{Análisis estadísticos}

\section{a) Resumen del modelo}

\begin{tabular}{|c|c|c|c|c|c|c|c|c|c|c|}
\hline \multirow[b]{2}{*}{ Modelo } & \multirow[b]{2}{*}{$\mathrm{R}$} & \multirow[b]{2}{*}{$\begin{array}{c}\mathrm{R} \\
\text { cuadrado }\end{array}$} & \multirow[b]{2}{*}{$\begin{array}{c}\mathrm{R} \\
\text { cuadrado }\end{array}$} & \multirow[b]{2}{*}{$\begin{array}{l}\text { Error estándar } \\
\text { de la estimación }\end{array}$} & \multicolumn{6}{|c|}{ Estadísticos de cambio } \\
\hline & & & & & $\begin{array}{l}\text { Cambio en } \\
\text { R cuadrado }\end{array}$ & $\begin{array}{l}\text { Cambio } \\
\text { en F }\end{array}$ & gl1 & gl2 & $\begin{array}{l}\text { Sig. } \\
\text { Cambio } \\
\text { en F }\end{array}$ & $\begin{array}{l}\text { Durbin- } \\
\text { Watson }\end{array}$ \\
\hline 1 & $.957 a$ & .916 & .910 & .29947 & .916 & 160.416 & 1 & 15 & .000 & 1.828 \\
\hline
\end{tabular}

a. Variable predictoras: (Constante), IIMUN

b. Variable dependiente: IDS

\section{b) Prueba KMO y Barlett}

\begin{tabular}{lcc|}
\hline $\begin{array}{l}\text { Kaiser-Meyer-Olkin de } \\
\text { adecuación de muestreo }\end{array}$ & .500 \\
\hline $\begin{array}{l}\text { Prueba de esfericidad } \\
\text { de Barlett }\end{array}$ & $\begin{array}{c}\text { Aprox. Chi- } \\
\text { cuadrado }\end{array}$ & 35.903 \\
\cline { 2 - 3 } & gl & 1 \\
\cline { 2 - 3 } & Sig. & .000 \\
\hline
\end{tabular}

\begin{tabular}{|c|c|c|c|c|c|c|c|c|c|c|c|c|c|c|}
\hline & \multirow{3}{*}{ Modelo } & \multirow{2}{*}{\multicolumn{2}{|c|}{$\begin{array}{l}\text { Coeficientes no } \\
\text { estandarizados }\end{array}$}} & \multirow{3}{*}{$\begin{array}{l}\text { Coef. } \\
\text { estanda- } \\
\text { rizados }\end{array}$} & \multirow{3}{*}{$\mathrm{t}$} & \multirow{3}{*}{ Sig } & \multirow{2}{*}{\multicolumn{2}{|c|}{$\begin{array}{c}95.0 \% \\
\text { intervalo de } \\
\text { confianza } \\
\text { para B }\end{array}$}} & \multicolumn{4}{|c|}{ Pruebas de Normalidad } & \multirow{2}{*}{\multicolumn{2}{|c|}{$\begin{array}{c}\text { Estadísticas } \\
\text { de } \\
\text { colinealidad }\end{array}$}} \\
\hline & & & & & & & & & \multicolumn{2}{|c|}{$\begin{array}{l}\text { Kolmogorov- } \\
\text { Smirnov }\end{array}$} & \multicolumn{2}{|c|}{ Shapiro-Wilk } & & \\
\hline & & B & Desv. & & & & 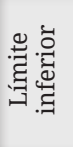 & 壳芯 & 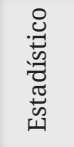 & Sig. & 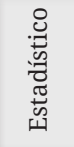 & Sig. & 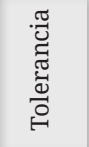 & VIF \\
\hline \multirow[t]{2}{*}{1} & (Constante) & -.542 & .084 & & -6.443 & .000 & .398 & .426 & .305 & .000 & .567 & 0.000 & 1.000 & 1.000 \\
\hline & IIMUN & .4 .384 & .343 & .957 & 12.783 & .000 & .316 & .888 & .300 & .000 & .526 & 0.000 & & \\
\hline
\end{tabular}

c) Coeficientes

\section{d) Ecuación de regresión I_IMUN \& I_DS}

\begin{tabular}{l|c|}
\hline \multicolumn{1}{c}{ Concepto } & Ecuación IDS: desarrollo sustentable \\
\hline $\begin{array}{l}\text { I_DS: Indicador de Desarrollo Sustentable } \\
\text { I_IMUN: Indicador de Ingresos Municipales }\end{array}$ & I_DS $=-0.542+4.384$ I_IMUN \\
\hline
\end{tabular}

Fuente: elaboración con apoyo del programa IBM SPSS Statistics V21 
En la tabla 3d se muestra la ecuación de regresión obtenida con los resultados de las tablas 3b y 3c. En ella se observa que durante el período analizado de 2000 a 2017 existe una correlación alta y significativa entre el indicador de ingresos municipales y el indicador de desarrollo sustentable, que manifiesta una alta relación entre ambas variables. Los resultados del análisis estadístico muestran que el coeficiente de correlación entre el índice de ingresos municipales y el índice de desarrollo sustentable es 0.957, con una significancia de 0.000 , lo que indica que existe una relación directamente proporcional entre la gestión pública y el desarrollo sustentable de las ciudades de Oaxaca (tabla 3a). Dicho de otra manera, entre mejor sea el manejo de los ingresos municipales, mayor será el desarrollo sustentable. Pero en la ecuación también se deduce que existen impedimentos para aumentar aún más los resultados en cuanto al desarrollo sustentable. Un parámetro que muestra esto es el valor negativo (-0.542) de la ecuación de regresión, que puede considerarse como una expresión de la debilidad de la capacidad institucional que padecen los gobiernos locales, particularmente en los aspectos administrativo, técnico y operativo.

\section{Discusión y conclusiones}

Teniendo como referencia los resultados descritos, y con respecto a la hipótesis de que durante el período 2000-2017 el uso de los ingresos municipales por parte de los gobiernos locales tiene una relación directamente proporcional con el desarrollo sustentable de las ciudades analizadas, se acepta, ya que existe una correlación alta y significativa entre ambas variables en la mayoría de las ciudades analizadas. Se destacan los casos de ciudades medianas, como Huatulco, Ixtlán, Puerto Escondido y Tlaxiaco, que a pesar de no tener un porcentaje alto de ingresos han logrado alcanzar un índice de desarrollo sustentable a la altura de aquellas grandes ciudades cuyos ingresos fueron más altos, como es el caso de la Zona Metropolitana de Oaxaca, la más importante en tamaño, infraestructura, política interna y como mayor centro cultural del estado de Oaxaca, que muestra un resultado relativamente desfavorable con respecto al resto de las ciudades. En resumen, existe un mejor desempeño en las grandes, medianas y pequeñas ciudades, que en las zonas metropolitanas. Esto último expone que el uso de los ingresos municipales no es el óptimo en todos los casos, para cumplir con las exigencias que las ciudades y sus habitantes demandan, teniendo con ello la posibilidad de plantear una línea de investigación que muestre por qué otros factores afectan la dinámica de desarrollo sustentable de las ciudades de mayor población del estado de Oaxaca. 
Es importante destacar que los resultados de la correlación de los índices descritos pueden ser considerados como aceptables pero no suficientes, ya que los niveles de ingresos municipales así como del desarrollo sustentable logrado alcanzan valores medios, lo que muestra déficits en lo económico, social y ambiental de las ciudades. A lo anterior se aúna el crecimiento poblacional al alza y mayores y más complejas problemáticas sociales que se visualizan, como por ejemplo la llegada de la crisis económica derivada del problema de salud mundial de la COVID-19, sin perder de vista que en el caso de México se considera al estado de Oaxaca dentro de los tres estados más pobres del país.

El escenario para el mejoramiento de la captación de ingresos y del desarrollo sustentable a corto plazo no es favorable para el estado al que pertenecen las ciudades analizadas, sobre todo porque el sector turístico es su mayor fuente de ingresos, y este como consecuencia de las restricciones de la pandemia tendrá un estancamiento. Por tal razón, más allá de cualquier estrategia en materia de política macroeconómica o política fiscal para afrontar la crisis, se debe considerar la creatividad que los propios ciudadanos puedan aportar para que la dinámica económica local no disminuya, aportando con ello un importante factor para que la economía del país resista los embates por la debilidad del sistema financiero y económico globalizado. Es decir, existen debilidades diversas en el aspecto social, económico, territorial, ambiental y político, que dificultan el mejoramiento del desarrollo sustentable. Por tal razón, resulta importante y urgente el fortalecimiento de los gobiernos locales, para que cuenten con la capacidad necesaria para planificar su territorio, utilizando sus fortalezas locales. En este sentido, independientemente de que los gobiernos locales de Oaxaca no cuenten con mucho presupuesto, tienen una alta potencialidad cultural, arquitectónica, turística-comercial y rural, y cuentan con la creatividad de sus ciudadanos para poder realizar el manejo adecuado de los ingresos municipales.

Finalmente, para que lo anterior se logre se necesita la implementación de una gestión local que destaque la inclusividad y la transparencia, capaz de convertir a las ciudades en oportunidades de desarrollo económico, social y ambientalmente equilibradas. Asimismo, se necesita la colaboración, sin condicionante alguno, de los niveles federal y estatal, ya que en estos niveles se encuentran la infraestructura, herramientas técnicas y capital humano necesarios para apoyar en las acciones del gobierno local y su comunidad. Dicho de otra manera, debe existir una preocupación para fortalecer las bases que sostienen el desarrollo sustentable de las ciudades, puesto que si estas cuentan con fortaleza, los tiempos de crisis 
serán menos perjudiciales. Se trata de reconocer que por muchos años no ha existido una preocupación real en lo local, siendo este nivel el que mayor aporte proporciona a la estabilidad de cualquier país, y que aún puede contribuir con la creatividad necesaria para convertir las oportunidades de la ciudad en posibilidades concretas de desarrollo nacional.

\section{Referencias}

BANCO MUNDIAL (2020). La COVID-19 (coronavirus) hunde a la economía mundial en la peor recesión desde la segunda guerra mundial. https://www. bancomundial.org/es/news/press-release/2020/06/08/covid-19-to-plungeglobal-economy-into-worst-recession-since-world-war-ii

CARMONA, N. \& CAAMAL, C. (2018). ¿Las transferencias federales han logrado reducir la desigualdad en las entidades federativas en México? EconoQuantum. 15(1), pp. 31 - 51.

CASADO, I. (2010). Apuntes sobre el origen y la historia de la ciudad. EUMEDNET. www. eumed.net/rev/cccss/07/icg2.htm

CONAPO (2017). Índices de intensidad migratoria México-Estados Unidos, 2000-2010. https://datos.gob.mx/busca/dataset/indice-absoluto-de-intensidadmigratoria-mexico--estados-unidos-2000--2010

CONAPO (2016). Datos abiertos del índice de marginación. http://www.conapo.gob.mx/es/ CONAPO/Datos_Abiertos_del_Indice_de_Marginacion

CONEVAL (2019). Indicadores de pobreza, pobreza por ingresos, rezago social y Gini a nivel municipal, 1990, 2000, 2005 y 2010. https://datos.gob.mx/busca/dataset/ indicadores-de-pobreza-pobreza-por-ingresos-rezago-social-y-gini-anivel-municipal1990-200-2010

COSUDE (2019). La buena gobernanza. https://www.eda.admin.ch/deza/es/home/cosude. html

DAMMERT, L. (2007). Seguridad pública en América Latina: ¿qué pueden hacer los gobiernos locales? Nueva Sociedad. (212), pp. 67 - 81.

ESPINOZA, C. (2017). Procesos etnopolíticos en la transición democrática chilena. Gobiernos locales y la vía política mapuche. Cuadernos de Antropología Social. (45), pp. 21-36.

FLORIDA, R. (2005). Cities and the Creative Class. Routledge.

GARCÍA, C. (2016). Teorías e historia de la ciudad contemporánea. Editorial Gustavo Gili, SL. 
GUTIÉRREZ, E. \& GONZÁLEZ, E. (2010). De las teorías del desarrollo al desarrollo sustentable: construcción de un enfoque multidisciplinario. Siglo XXI.

HERNÁNDEZ, E.; ADAME, S. \& CADENA, E. (2017). Los retos de la sustentabilidad urbana en México. Reflexiones sobre su evaluación a través de la Metodología. Quivera, 19(1), pp. 85-97.

INAFED (2019). Principales datos socioeconómicos por municipio. http://www.inafed.gob. $\mathrm{mx} / \mathrm{es} /$ inafed/Principales_Datos_Socioeconomicos_por_Municipio

INEGI (2019a). Estadísticas de finanzas públicas estatales y municipales. https://www.inegi. org.mx/sistemas/olap/proyectos/bd/continuas/finanzaspublicas/fpmun. asp?s=est\&c=11289\&proy=efipem_fmun

INEGI (2019b). Banco de Información Económica. https://www.inegi.org.mx/sistemas/bie/

IONITA, L.; RADU, F. \& TABIRCA, L. (2011). Needs of Local Sustainable Development. Annals of Faculty of Economics. 1(2), pp. 91-97.

KEN, C. A. (2014). Desarrollo Regional y gestión pública en Quintana Roo 1970 a 2010. Estudio de caso municipio de Othón Blanco. MA Porrúa.

LÓPEZ, R. \& CHAUCA, P. (2010). Iniciativas de gobierno en México para el desarrollo local sustentable. La experiencia de innovación en Michoacán. En: Amozurrutia, J. A., R. Mansilla y J. Matus (Coords.) Jornadas Anuales de Investigación 2009. Centro de Investigaciones Interdisciplinarias en Ciencias y Humanidades, UNAM.

LÓPEZ, R.; AYALA, D. \& ARELLANES, Y. (2017). Gobernabilidad democrática y desarrollo local sustentable. Economía y Sociedad. 21(36), pp. 61-75.

LÓPEZ, C. \& SORIA, R. (2013). Alternancia política y nueva gestión pública: un análisis comparativo, 1998-2008. Región y sociedad. 25(56), pp. 1870-3925.

LÓPEZ, M.; MIGUEL, A. \& MARTÍNEZ, K. (2018). Ciudades: análisis de sus desigualdades inter e intraurbanas. Bitácora Urbano-Territorial. 28(3), pp. 27-38.

MARTÍNEZ, G. K. (2018). La vivienda en el desarrollo sustentable de las pequeñas, medianas y grandes ciudades de Oaxaca. Eumed.

MIGUEL, A. (2012). El Desarrollo Sustentable y el Cambio Climático en las Regiones de México: el caso del Sur-sureste, periodo 2000-2010. Secretaría de Educación Pública. Subsecretaría de Educación Superior. Instituto Tecnológico de Oaxaca.

MOUTINHO, M. \& FARIA, A. (2019). Local development versus neoliberal globalization project: reflecting on market-oriented cities. Revista de Administración Pública. 53(1), pp. 84 - 100. 
PÉREZ, E. \& ARENAS, E. (2012). Agenda desde lo Local: Desarrollo Sostenible y Desarrollo Humano. Revista Austral de Ciencias Sociales. (22), pp. 43-54.

PNUD (2014). Índice de desarrollo humano municipal en México: Nueva Metodología. Programa de las Naciones Unidas para el Desarrollo.

SALVATTO, F. \& BANZATO, G. (2013). Poder y gobierno local en México, 1808-1857. Instituto de Investigaciones en Ciencias Sociales y Humanidades. (24), pp. 463-467.

SÁNCHEZ, A., ROSAS, J. y GARCÍA, M. (2018). La evolución de la investigación sobre los gobiernos municipales en México, 1984-2016. Revista mexicana de ciencias políticas y sociales. 63(232), pp. 45-75.

SÁNCHEZ, M. \& MARTÍNEZ, H. (2016). Gobierno local abierto: diagnóstico en México y Oaxaca. RICSH Revista Iberoamericana de las Ciencias Sociales y Humanísticas. 5(10), pp. 1-35.

SEFIN (2018). Aportaciones y participaciones municipales. https://www.finanzasoaxaca. gob.mx/aportacion-municipal/

SEPÚLVEDA, S. (2008). Metodología para estimar el nivel de desarrollo sostenible de territorios. San José, Costa Rica: Instituto Interamericano de Cooperación para la Agricultura (IICA).

SESNSP (2020). Incidencia delictiva. México. https://www.gob.mx/sesnsp/acciones-y-programas/incidencia-delictiva-87005?idiom=es

SCOTT, A. (2007). ¿Capitalismo y urbanización en una nueva clave? la dimensión Cognitivo-Cultural. Tabula Rasa. (6), pp. 195-217.

TORÍBIO, A. (2013). Project “smart cities": some remarks about efficiency and sustainability. Revista del CESLA. (16), pp. 69-80.

URIBE, M. (2020). La calidad como objetivo. Propuesta metodológica. Bitácora Urbano-Territorial. 30 (1), pp. 167-179.

VARELA, E. (2015). Nuevos roles de los gobiernos locales en la implementación de políticas públicas. Gobernabilidad y Competitividad global. EURE. 41(123), pp. 213-237. 



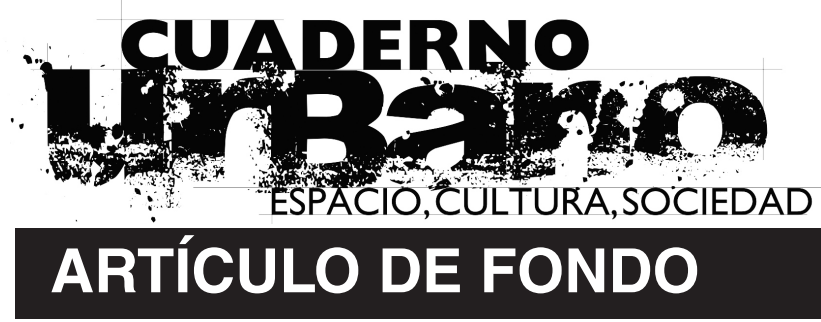


